\title{
In-situ TEM study of thin-film vanadium oxide stability
}

\author{
B.D. Gauntt,* E.C. Dickey**
}

* Materials Science and Engineering Department, Pennsylvania State University, University Park, PA 16802

** Materials Research Institute, Pennsylvania State University, University Park, PA 16802

Vanadium oxide thin films were co-deposited via reactive pulsed-dc sputtering onto both formvarcoated $\mathrm{Cu}$ TEM grids and $\mathrm{SiO}_{2}$-coated $\mathrm{Si}$ wafers. A trend was observed throughout the series whereby increasing oxygen partial pressure in the growth chamber caused an increase in both the oxygen content and the disorder in the films [1]. Films deposited below a $\mathrm{PO}_{2}$ of $5 \%$ exhibited the presence of nanocrystals and films deposited above a $\mathrm{PO}_{2}$ of 5\% were amorphous. Two films from the series, one nano-crystalline with an amorphous component, and the other completely amorphous, were found to have the same stoichiometry via rutherford backscattering spectroscopy. Though the bulk stoichiometry of both films was found to be $\mathrm{VO}_{2}$, the nanocrystalline phase was determined to be the FCC phase, which at equilibrium is characteristic of $\mathrm{VO}_{\mathrm{x}}$, where $0.8<\mathrm{x}<1.3$.

The $\mathrm{VO}_{2}$ (stoichiometry) films were subjected to annealing in the transmission electron microscope at $350^{\circ} \mathrm{C}$ with in-situ observation by both bright-field imaging and electron diffraction, shown in Fig 1. The amorphous film with and $\mathrm{O}: \mathrm{V}$ ratio of $2: 1$ transformed to rutile $\mathrm{VO}_{2}$, as expected from the equilibrium phase diagram. The nano-crystalline film with an $\mathrm{O}: \mathrm{V}$ ratio of $2: 1$ did not transform to crystalline $\mathrm{VO}_{2}$, but remained as $\mathrm{FCC} \mathrm{VO}_{\mathrm{x}}$; however, a diffuse low angle scattering feature moved to higher scattering angle. Finally, a nano-crystalline film with an $\mathrm{O}: \mathrm{V}$ ratio of $0.8: 1$ remained as FCC $\mathrm{VO}_{\mathrm{x}}$, as would be expected, and the presence of a diffuse low-angle feature disappeared upon annealing.

Electron energy loss spectroscopy (EELS) was used to characterize the films both before and after annealing. EEL spectra from the nano-crystalline films before and after annealling are essentially indistinguishable. EELS of the partly-crystallized amorphous film shows the presence of two distinct spectra, one from crystalline regions and one from the amorphous regions, see Figs 3 and 4. The main difference between the two spectra is the presence of near-edge and post-edge structure in the O-k edge. The first difference is a "splitting" of the O-K edge into two distinct peaks. This same near-edge structure is also observed in spectra obtained from $\mathrm{VO}_{2}$ reference powder. The second distinction between the amorphous and crystalline regions is the presence of an additional feature around $560 \mathrm{eV}$ energy loss, which is found in crystalline spectra and lacking in amorphous spectra. The $\mathrm{O} \mathrm{K}$ near edge split can be interpreted in terms of crystal field theory [3], and results from degeneracy created in hybridized $\mathrm{O} 2 \mathrm{p} / \mathrm{V} 3 \mathrm{~d}$ orbitals due to interaction between the $\mathrm{V}$ states and the octahedrally coordinated $\mathrm{O}$ atoms.

Interestingly, the amorphous film that transformed to rutile $\mathrm{VO}_{2}$ lacks a metal-to-insulator transition (MIT), which is commonly found in $\mathrm{VO}_{2}$ [2], and results from a phase transformation at $68{ }^{\circ} \mathrm{C}$ from a monoclinic structure to the high-temperature rutile structure. The constrained thin-film geometry combined with the residual amorphous phase constrains the film in such a way that the MIT cannot occur. Annealing at $350{ }^{\circ} \mathrm{C}$ caused the resistivity of both $\mathrm{VO}_{2}$ films to increase; however the thermal coefficient of resistance (TCR) was left unchanged for the nano-crystalline $\mathrm{VO}_{\mathrm{x}}$ film while 
the TCR increased for the film that partially transformed to rutile $\mathrm{VO}_{2}$. It is evident that post process annealing affects both the microstructure and consequently the electrical properties. Future work will focus on elucidating a direct relationship between local atomic order and both resistivity and TCR.

\section{References}

[1] B.D. Gauntt et al., J. Mat. Res. Accepted for Publication (2009).

[2] D.B. McWhan et al., Phys. Rev. B. 10(1974) 490.

[3] M. Willinger, Investigation of the Oxygen K-Edge Fine Structure in Vanadium Oxides, Ph.D. Thesis, TU Wien, 2001.

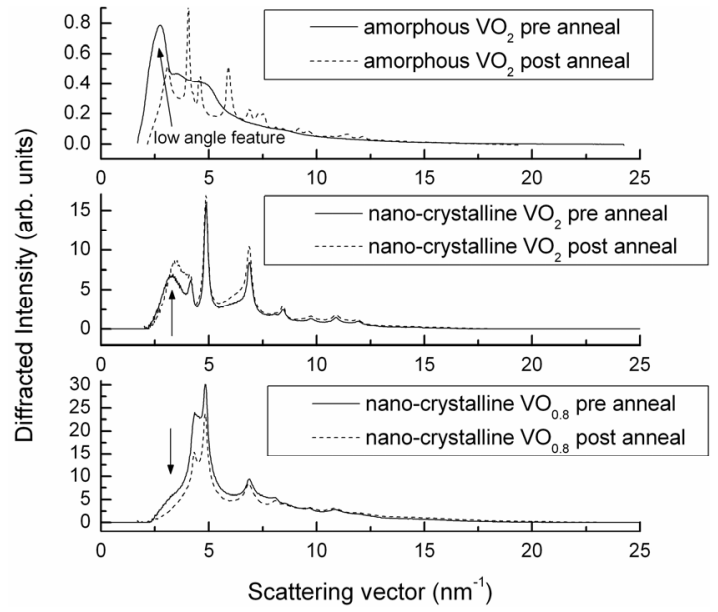

Fig 1. Diffracted intensity of both amorphous (5 mTorr $10 \%)$ and nano-crystalline $(2.5$ and 5 mTorr 5\%) films before and after in-situ anneal.

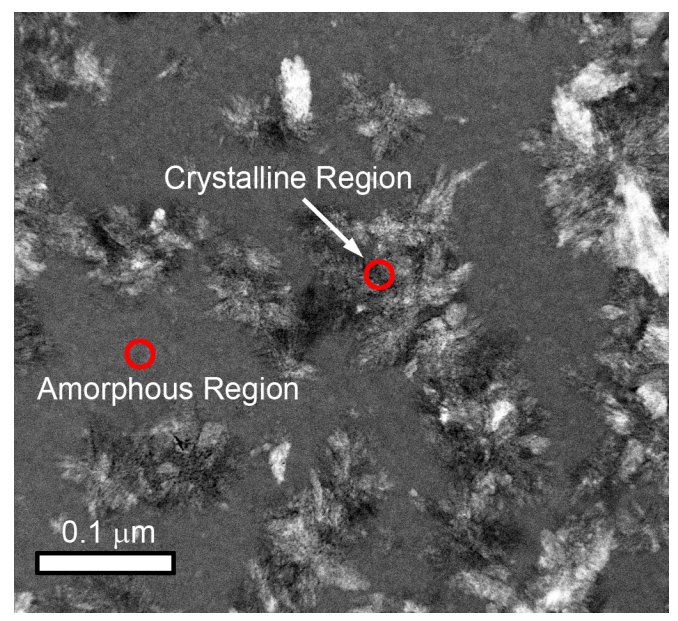

Fig 3. ADF STEM image of partiallycrystallized amorphous film showing regions used for EELS spectra shown in Fig 4.

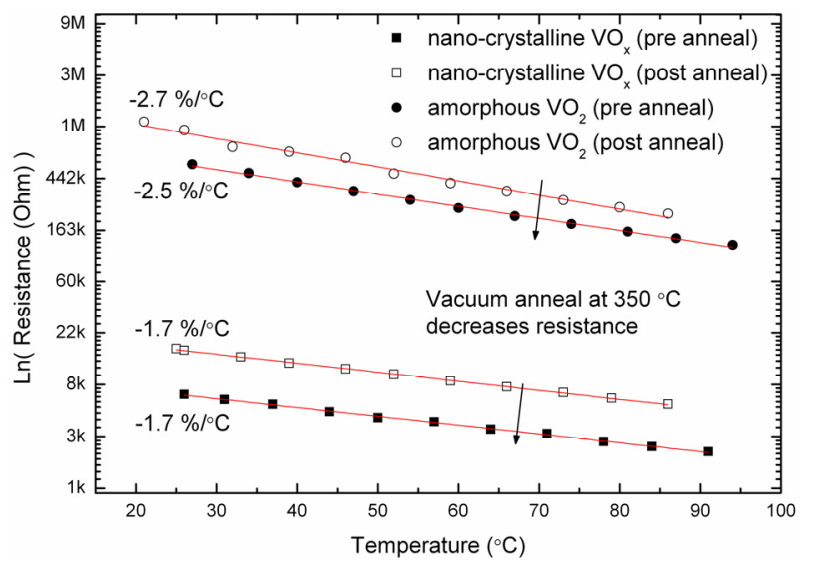

Fig 2. Resistance of amorphous film and nanocrystalline film before and after anneal as a function of sample temperature.

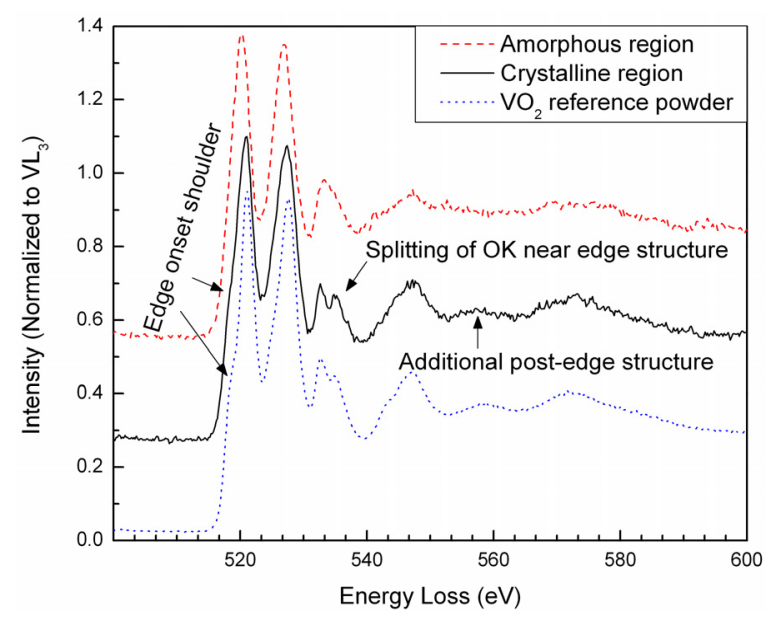

Fig 4. EELS spectra showing $\mathrm{V} \mathrm{L}_{2,3}$ edge and $\mathrm{O}$ $\mathrm{K}$ edge. Several differences between amorphous areas and crystalline regions are evident in the $\mathrm{O}$ $\mathrm{K}$ near and post-edge regions. 